\title{
Localization of aquaporin-3 proteins in the bovine rumen
}

\author{
Chongliang Zhong, (1) Alan Farrell, and Gavin S. Stewart* (이 \\ School of Biology and Environmental Science, Science Centre West, University College Dublin, Belfield, Dublin 4, Ireland
}

\begin{abstract}
Urea nitrogen salvaging is a crucial mechanism that ruminants have evolved to conserve nitrogen. Facilitative urea transporter-B proteins are known to be involved in urea transport across the rumen epithelium and thus efficiently facilitate the urea nitrogen salvaging process. Recently, functional studies have suggested that aquaglyceroporin transporters might also play a significant role in ruminal urea transport and aquaporin-3 (AQP3) protein has previously been detected in rumen tissue. In this current study, we investigated the specific localization of AQP3 transporters in the bovine rumen. First, end-point reverse-transcription PCR experiments confirmed strong AQP3 expression in both bovine rumen and kidney. Immunoblotting analysis using 2 separate anti-AQP3 antibodies detected AQP3 protein signals at 25,32 , and $42-45 \mathrm{kDa}$. Further immunolocalization studies showed AQP3 protein located in all the layers of rumen epithelium, especially in the stratum basale, and in the basolateral membranes of kidney collecting duct cells. These data confirm that AQP3 transporters are highly abundant within the bovine rumen and appear to be located throughout the ruminal epithelial layers. The physiological significance of the multiple AQP3 proteins detected and their location is not yet clear, hence further investigation is required to determine their exact contribution to ruminal urea transport.
\end{abstract}

Key words: aquaporin-3, urea transporter-B, bovine, rumen

\section{INTRODUCTION}

The symbiotic relationship with the microbial populations that reside in the rumen is crucial to all ruminants. This relationship is supported by the urea nitrogen salvaging (UNS) process, in which urea is secreted into the rumen and is used by bacteria as a

Received October 11, 2019.

Accepted November 29, 2019.

*Corresponding author: gavin.stewart@ucd.ie nitrogen source (Stewart and Smith, 2005). By supporting continued bacterial growth in this manner, ruminants benefit as they (1) can assimilate the produced microbial proteins, and (2) use the short-chain fatty acids produced by bacterial fermentation processes as a vital energy source (Stewart and Smith, 2005). The extent of UNS depends on the efficiency of urea transport from the blood into the rumen, with current research emphasis investigating the molecular basis for this process (Stewart et al., 2005).

Since the identification of facilitative urea transporters (UT) in mammals, they have been the prime candidates for transporting urea in UNS. For example, UT-B2 was found to be abundantly expressed in the rumen epithelium and facilitates urea transport across the rumen in a phloretin-sensitive and diet-induced manner (Stewart et al., 2005; Simmons et al., 2009; Berends et al., 2014). However, more recently, functional studies have shown that other mechanisms might also contribute, as a variable portion of urea flux (up to $50 \%$ ) was not inhibited by the UT inhibitor, phloretin (Walpole et al., 2015). Specifically, the aquaglyceroporins (AQGP), a group of membrane proteins in the aquaporin (AQP) family that mainly transport glycerol and water across membranes, are thought to be an alternative urea transport mechanism (Røjen et al., 2011). In vitro studies have shown that AQGP (AQP3, AQP7, AQP9, and AQP10) are permeable to urea (Rojek et al., 2008) and that many of these AQGP transcripts have been found in rumen papillae (Røjen et al., 2011; Walpole et al., 2015). Interestingly, previous studies have reported that ruminal urea transport is $\mathrm{pH}$ regulated (Abdoun et al., 2010; Lu et al., 2014) and AQP3 is known to also be modulated by pH (Zeuthen and Klaerke, 1999). Crucially, adding the AQPinhibitor $\mathrm{NiCl}_{2}$ markedly decreased urea flux (serosalto-mucosal) by $23 \%$ across the rumen (Walpole, et al., 2015), in addition to any effects of phloretin, strongly indicating that AQGP play a functional role in ruminal urea transport. The exact regulatory mechanisms, however, are still unclear. Røjen et al. (2011) showed that transcript expression of AQP3, AQP7, and AQP10 was greater in high-nitrogen-fed cows compared with low-nitrogen-fed cows, whereas the abundance of AQP3 
Table 1. Primers used in end point reverse-transcription $\mathrm{PCR}^{1}$

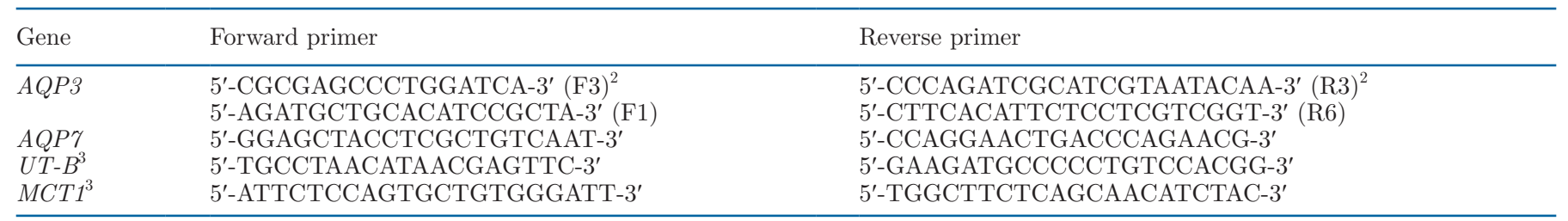

${ }^{1} \mathrm{~F}(\mathrm{n})=$ forward primer against exon $(\mathrm{n}) ; \mathrm{R}(\mathrm{n})=$ reverse primer against exon $(\mathrm{n})$.

${ }^{2}$ Taken from Røjen et al., 2011.

${ }^{3}$ Taken from Coyle et al., 2016.

and AQP7 protein was not affected by dietary nitrogen concentration. None of these, however, correlated with the increased rumen epithelial urea permeability observed with low dietary nitrogen concentration in the same study (Kristensen et al., 2010). In terms of energy regulation, Walpole et al. (2015) observed that ruminal serosal-to-mucosal urea flux tended to increase linearly as calves were adapted to diets with increased fermentability (from a hay diet to hay-based mediumgrain diet). In line with this, linear increases of AQP3 and UT-B transcript expression were also observed, whereas no significant responses occurred for AQP7 and AQP10. Other studies have shown an increase in AQP3 and UT-B transcripts with the addition of solid feed to the diets of milk-fed calves (Berends et al., 2014), and a decrease in AQP3 expression in the rumen of fattening bulls when urea was introduced in the diet (Saccà et al., 2018). However, most of the work has been performed at a gene transcription level, while protein abundance has seldom been investigated.

As far as can be discerned, Røjen et al. (2011) is the only published study that investigated AQGP protein abundance in the rumen. Therefore, more detailed experiments are needed to comprehensively characterize the abundance and localization of AQGP. As stated, various studies (Røjen et al., 2011; Berends et al., 2014; Walpole et al., 2015; Saccà et al., 2018) have previously indicated AQP3 transcription is more responsive to diets. Therefore, in this present study we investigated the expression, abundance and localization of AQP3 transporters in the bovine rumen.

\section{MATERIALS AND METHODS}

Tissue samples were excised from dorsal, ventral, and cranial sacs of adult Holstein-Friesian cattle rumen obtained in a commercial slaughterhouse within $1 \mathrm{~h}$ of slaughter. This was part of our ongoing, long-term study into the transport mechanisms involved in the UNS process in cattle, which has already characterized UT-B urea transporters along the gastrointestinal tract (Coyle et al., 2016). Total RNA of the rumen papillae was extracted using a commercial kit according to manufacturer instructions (RNA-STAT 60, AMS Biotechnology, Milton, UK). Total RNA of bovine kidney was purchased from AMS Biotechnology. For both rumen and kidney, $1 \mu \mathrm{g}$ of total RNA was reverse transcribed via reverse transcriptase (SensiFAST reverse transcription kit, Bioline, Nottingham, UK). The resulting material, an untranscribed control sample or $\mathrm{H}_{2} \mathrm{O}(1 \mu \mathrm{L})$ then underwent PCR amplification with a Taq polymerase (GoTaq Hot Start Master Mix, Promega, Madison, WI) using primer pairs specific for each gene (see Table 1). Cycling parameters included an initial denaturation step at $94^{\circ} \mathrm{C}$ for $2 \mathrm{~min}$, followed by 35 cycles of $94^{\circ} \mathrm{C}$ for $30 \mathrm{~s}, 53^{\circ} \mathrm{C}$ or $60^{\circ} \mathrm{C}$ for $30 \mathrm{~s}$, and $72^{\circ} \mathrm{C}$ for $1 \mathrm{~min}$. A final extension step of $72^{\circ} \mathrm{C}$ for 10 min was also included. PCR products were run on $1 \%$ agarose gels and imaged under a UV imager (SYNGENE, UK). Target bands were excised, purified (GeneClean Turbo kit, MP Biomedicals, Irvine, CA), and identified by direct sequencing (Eurofins MWG, Ebersberg, Germany).

For membrane-enriched protein samples, rumen papillae or human RT4 urothelial cells were homogenized in an ice-cold buffer (12 $\mathrm{m} M$ HEPES, $300 \mathrm{~m} M$ mannitol, $\mathrm{pH}$ 7.6) and centrifuged at 2,500 $\times g$ for $10 \mathrm{~min}$ at $4^{\circ} \mathrm{C}$. The resulting supernatant was collected and centrifuged at $17,000 \times g$ for another $30 \mathrm{~min}$ at $4^{\circ} \mathrm{C}$. The resulting plasma membrane-enriched pellets were retained and re-suspended in homogenization buffer. Deglycosylation analysis was undertaken by pre-incubating protein samples for $2 \mathrm{~h}$ at $37^{\circ} \mathrm{C}$ with or without the presence of peptide: N-glycosidase F (PNGase F) enzyme (New England Biolabs, Ipswich, MA). Laemmli sample buffer $(2 \times$, Sigma, St. Louis, MO) with reducing agent was added to protein samples before being heated at $70^{\circ} \mathrm{C}$ for $15 \mathrm{~min}$. The SDS-PAGE was performed on 8 to $16 \%$ pre-cast gels (Bio-Rad, Hercules, CA) by loading $\sim 20 \mu \mathrm{g} /$ lane of membrane-enriched samples. After being transferred to nitrocellulose membranes (Bio-Rad), the immunoblots were blocked with $5 \%$ nonfat dry milk in washing buffer $(25 \mathrm{mM}$ Tris, $192 \mathrm{mM}$ glycine, $0.2 \%$ Tween 20 ) for $2 \mathrm{~h}$ at room temperature, 
and probed overnight at $4^{\circ} \mathrm{C}$ with 1 of 2 anti-AQP3 antibodies; 1:1,000 AQP3-HPA (HPA014924, Sigma; QLMIGCHLEQPPPSNEEENVKLAHVKHKEQI target sequence of the last $31 \mathrm{AA}$ in the $\mathrm{C}$-terminus of human AQP3) or 1:1,000 AQP3-SAB (SAB5200111, Sigma; undisclosed target sequence within C-terminus of rat AQP3). After the primary antibody, immunoblots were washed and probed with 1:5,000 horseradish peroxidase-linked anti-rabbit secondary antibody for 1 $\mathrm{h}$ at room temperature. After another wash, detection of protein was performed using the Western Lightning Plus ECL reagents (Perkin Elmer, Waltham, MA) and an image reader (LAS4000, Fujifilm, Tokyo, Japan).

Paraformaldehyde-fixed, paraffin-embedded bovine rumen (Coyle et al., 2016) and kidney sections (AMS Biotechnology) were used for immunohistochemistry experiments. After paraffin removal and rehydration in a decreasing concentration series of ethanol (100 to $70 \%$ ), sections were incubated in $0.3 \%$ hydrogen peroxide in methanol to block endogenous peroxidase. Antigen retrieval was performed by heating sections in $99^{\circ} \mathrm{C}$ water bath for 2 min (rumen) or in a microwave on full power $(900 \mathrm{~W})$ for $5 \mathrm{~min}$ (kidney) in a buffer containing $25 \mathrm{~m} M$ Tris-HCl $(\mathrm{pH} 8.0)$ and $10 \mathrm{~m} M$ EDTA. After cooling down, sections were incubated overnight with 1:250 (rumen) or 1:100 (kidney) of anti-AQP3 antibodies, or 1:500 (rumen) or 1:100 (kidney) of UT-B antibody (Walpole et al., 2014; Coyle et al., 2016) diluted in antibody solution (0.1\% BSA and $0.3 \%$ Triton X-100 in PBS). For peptide incubation experiments, primary antibody was pre-incubated with a specific immunizing peptide ( $1 \mu \mathrm{g}$ of peptide/ $\mu \mathrm{L}$ of antibody) or an equivalent amount of a nonspecific peptide for $24 \mathrm{~h}$ using a rotating mixer. Immunostaining was visualized with a 1:200 diluted horseradish peroxidase-linked antirabbit secondary antibody followed by incubation with diaminobenzidine (Sigma) and counterstaining with hematoxylin. Stained sections were then dehydrated in an increasing concentration series of ethanol (70-100\%) and treated with Neo-clear (Millipore, Darmstadt, Germany). Finally, sections were mounted with coverslips using a Eukitt (Sigma-Aldrich, Darmstadt, Germany) mounting medium and imaged using a DMLB microscope, DFC420C digital camera, and Application Suit V4 software (Leica, Wetzlar, Germany).

\section{RESULTS AND DISCUSSION}

Initial end-point reverse-transcription PCR experiments were performed to investigate ruminal mRNA AQP3 expression. Primer set AQP3-F3R3, targeted to exon 3 of bovine AQP3 and based on primers used by Røjen et al. (2011), detected strong signals both in the dorsal and ventral sac of rumen (Figure 1A). In con- trast, a set of AQP7 primers against exon 4 and exon 5 detected only moderate AQP7 expression (Figure 1A). Previously characterized primers for UT-B2 and monocarboxylate transporter 1 (MCT1) were used to test sample integrity and gave the expected results of strong UT-B2 and MCT1 signals, with a weak UT-B1 signal

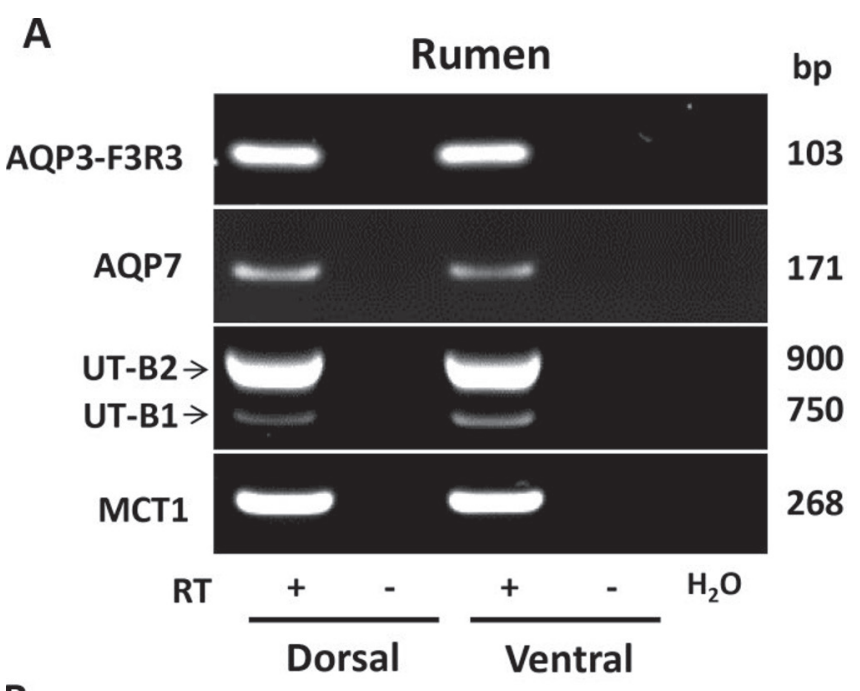

B

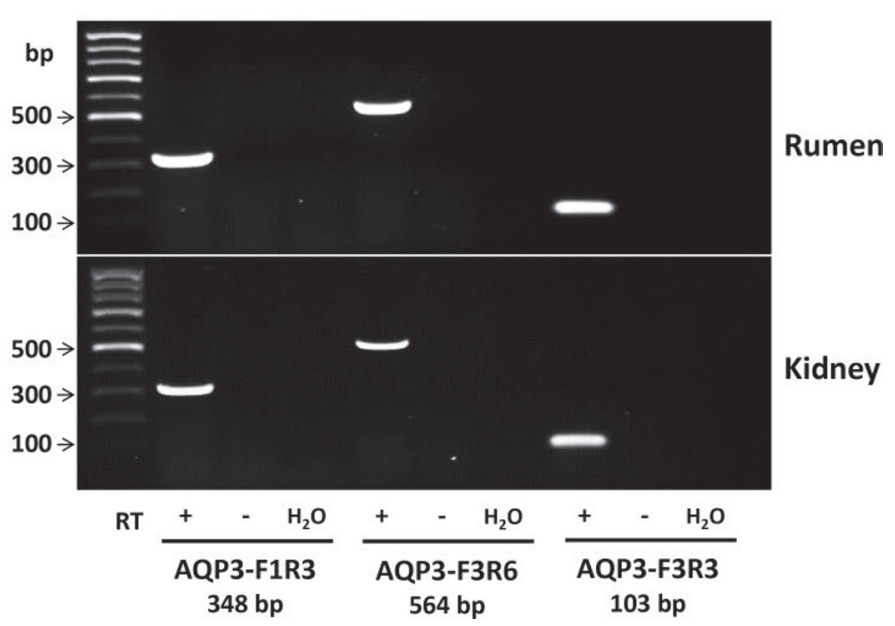

Figure 1. End point reverse-transcription (RT) PCR experiments showing gene expression in the bovine rumen and kidney. (A) Strong signals at the predicted $103 \mathrm{bp}$ were detected by aquaporin-3 (AQP3)F3R3 primers in both dorsal and ventral sacs of the rumen, whereas moderate signals at the predicted $171 \mathrm{bp}$ were detected by AQP7 primers. Isoform-specific urea transporter (UT)-B primers detected strong 900-bp UT-B2 signals and weak 750-bp UT-B1 signals in both the dorsal and ventral rumen. Strong signals were also detected for monocarboxylate transporter 1 (MCT1) at the predicted $268 \mathrm{bp}$. (B) The primer set targeted against exon 1 and exon 3 of AQP3 (AQP3F1R3) detected a single band at the predicted 348 bp both in the rumen and kidney. Similarly, primers against exon 3 and exon 6 (AQP3F3R6) gave a single band at the predicted $564 \mathrm{bp}$. No alternatively spliced transcripts were detected. Again, a strong 103-bp signal was detected by AQP3-F3R3 primers. + = RT present; $-=\mathrm{RT}$ absent; $\mathrm{F}(\mathrm{n})=$ forward primer against exon $(\mathrm{n}) ; \mathrm{R}(\mathrm{n})=$ reverse primer against exon $(n)$. 
(Coyle et al., 2016). Overall, the abundant AQP3 transcript expression agrees with previous studies (Røjen et al., 2011; Saccà et al., 2018) and suggests that AQP3 may play an important role in bovine rumen function. To test for alternatively spliced AQP3 transcripts, we designed a forward primer against exon 1 (F1) and a reverse primer against exon 6 (R6). By combining these in experiments with the AQP3-F3R3 primers, we obtained another 2 primer sets that amplified from exon 1 to exon 3 (AQP3-F1R3) and exon 3 to exon 6 (AQP3-F3R6). Neither of these new primer sets detected alternatively spliced transcripts, with only single PCR products of the predicted size being produced for both bovine rumen and kidney samples (Figure 1B). Finally, all 3 of these rumen PCR products were confirmed to be bovine AQP3 by direct sequencing (data not shown).

To confirm the viability of the 2 commercial antiAQP3 antibodies, AQP3-HPA and AQP3-SAB, immunoblotting analysis was first performed using hu- man RT4 urothelial cells, which had previously been shown to abundantly express AQP3 (Rubenwolf et al., 2014). For AQP3-HPA, deglycosylation treatment with PNGase F enzyme partially shifted a 42 to $45 \mathrm{kDa}$ smeared signal to a core $25-\mathrm{kDa}$ protein. For AQP3$\mathrm{SAB}$, although there was higher background staining, the same deglycosylated $25-\mathrm{kDa}$ signal was observed (Figure 2A). In contrast, similar experiments with bovine rumen protein revealed slightly different results. Whereas AQP3-SAB again detected $42-45$ and $25 \mathrm{kDa}$ signals, AQP-HPA detected unglycosylated protein at $32 \mathrm{kDa}$ (Figure 2B).

Aquaporin-3 is a glycoprotein and studies of the transporter usually detect both glycosylated and unglycosylated proteins. For example, both $45-\mathrm{kDa}$ glycosylated and $25-\mathrm{kDa}$ unglycosylated proteins are reported in human red blood cells (Roudier et al., 2002). Similarly, 35-44 and $26 \mathrm{kDa}$ AQP3 proteins are found in rat ureter and bladder (Spector et al., 2002), whereas 33-40 and $27 \mathrm{kDa}$ have been reported
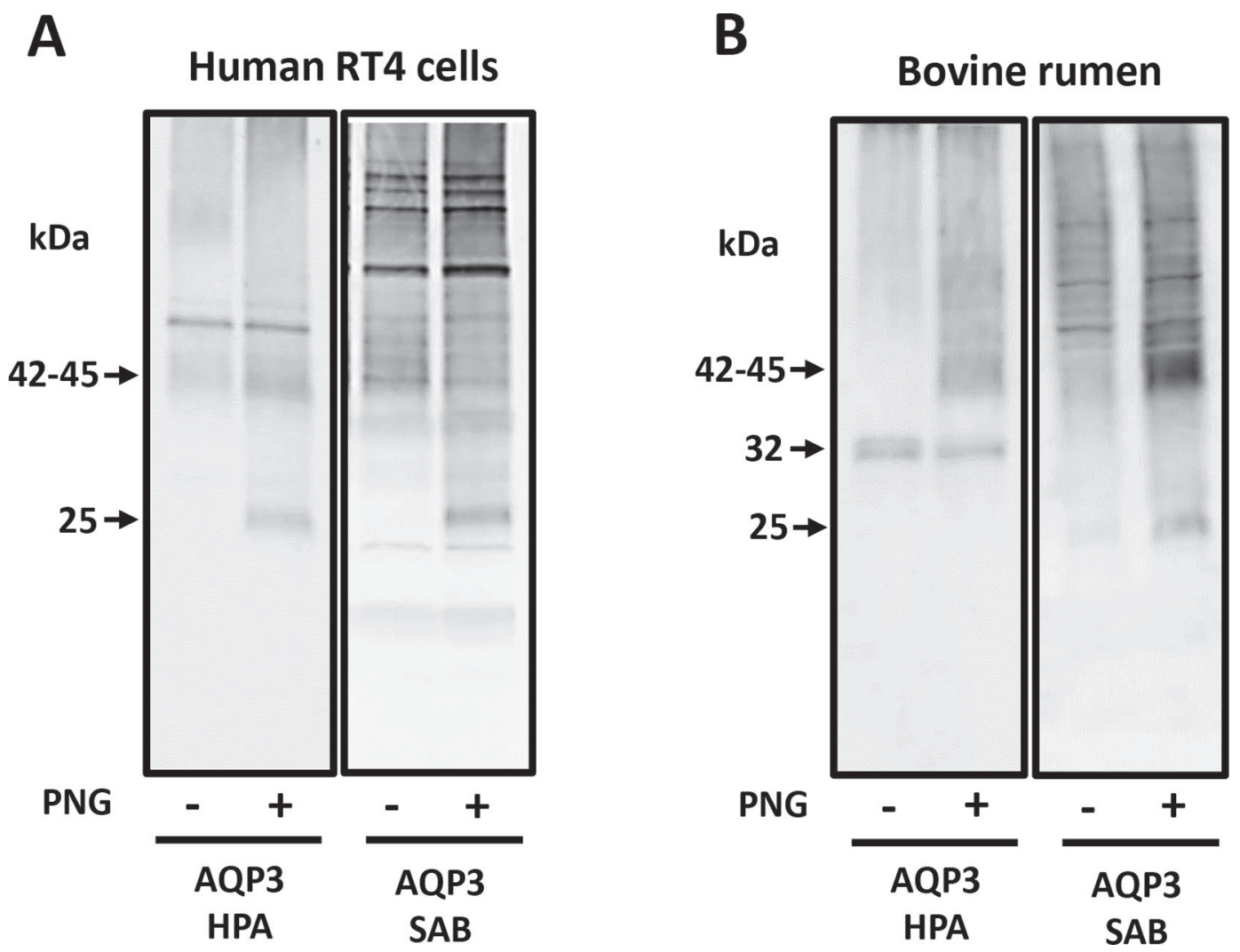

Figure 2. Immunoblots showing aquaporin-3 (AQP3) protein abundance in human RT4 urothelial cells and bovine rumen. Two different anti-AQP3 antibodies, AQP3-HPA (HPA014924, Sigma, St. Louis, MO) and AQP3-SAB (SAB5200111, Sigma), were used to detect bovine AQP3 protein. (A) Using membrane-enriched fractions of RT4 cells, AQP3-HPA detected a smeared signal of 42-45 kDa, which could be partially shifted to $25 \mathrm{kDa}$ by PNGase F (New England Biolabs, Ipswich, MA) treatment. Although more background staining was apparent, AQP$\mathrm{SAB}$ also detected the $25 \mathrm{kDa}$ signal in deglycosylated RT4 protein. (B) Using rumen cell membrane-enriched samples (dorsal sac), AQP3-HPA detected a strong, unglycosylated signal at $32 \mathrm{kDa}$, as well as the $42-45 \mathrm{kDa}$ smear. In contrast, rumen protein AQP3-SAB again detected the 25 and $42-45$ kDa signals. PNG $=$ PNGase F enzyme; + = PNGase F with buffers; $-=$ buffers only. 
in rat kidney (Ecelbarger et al., 1995). We therefore believe that the $42-45$ and $25 \mathrm{kDa}$ signals we detected with AQP3-SAB in bovine rumen represent the standard glycosylated and unglycosylated bovine AQP3. Intriguingly, the $32 \mathrm{kDa}$ core protein that AQP3-HPA detected in bovine rumen is exactly the same size as the $32-\mathrm{kDa}$ protein recently reported in $\mathrm{HaCaT}$ cells (i.e., immortalized human skin keratinocytes), which was confirmed to be AQP3 protein by genetic silencing (Martinotti et al., 2019). We therefore believe that the rumen $32 \mathrm{kDa}$ protein is likely to represent an alternative isoform of the bovine AQP3 core protein. Since our PCR analysis found no evidence of alternatively spliced transcripts (Figure 1B), this protein may actually be derived from an alternative translation initiation site. This concept was first discussed by Ecelbarger et al. (1995) 25 yr ago when they were attempting to explain the discrepancy between observed and predicted sizes for rat AQP3 protein. Basically, they stated that if the translation was initiated from the first methionine site, a 292 AA protein (predicted size of $31.4 \mathrm{kDa}$ ) would be produced. In contrast, if the translation was started further downstream at the fourth methionine site, a $256 \mathrm{AA}$ protein (predicted size of $27.2 \mathrm{kDa}$ ) would be produced. However, further experiments are required to confirm whether or not this explains the 2 AQP3 protein signals detected in this current study. For example, future studies could utilize antibodies targeted to the first 20 to $25 \mathrm{AA}$ in the $\mathrm{N}$ terminus of the proposed, longer 292 AA protein.

In the final set of experiments, we carried out immunohistochemistry to localize AQP3 protein in bovine rumen and kidney. Strong signals were detected in the bovine kidney with both AQP3 antibodies, specifically at the basolateral membrane of collecting duct cells (Figure 3). In contrast, no such signals were observed for UT-B. These data were consistent with the known location of AQP3 in the mammalian kidney (Ecelbarger et al., 1995). Intriguingly, in the rumen, AQP3-HPA staining (presumably of $32 \mathrm{kDa}$ protein) was distributed throughout all the layers of rumen epithelium, namely in the stratum basale, stratum spinosum, stratum granulosum, and surprisingly, stratum corneum (Figure 3). In contrast, AQP3-SAB staining (presumably of $25 \mathrm{kDa}$ protein) was mainly found in the stratum basale, in a pattern very similar to that observed for UT-B (Figure 3). The AQP3 distribution in stratum corneum was somewhat unexpected as no tight junctions are found in that layer (Graham and Simmons, 2005). However, the AQP3-HPA signals

\section{AQP3-HPA}

AQP3-SAB

UT-B

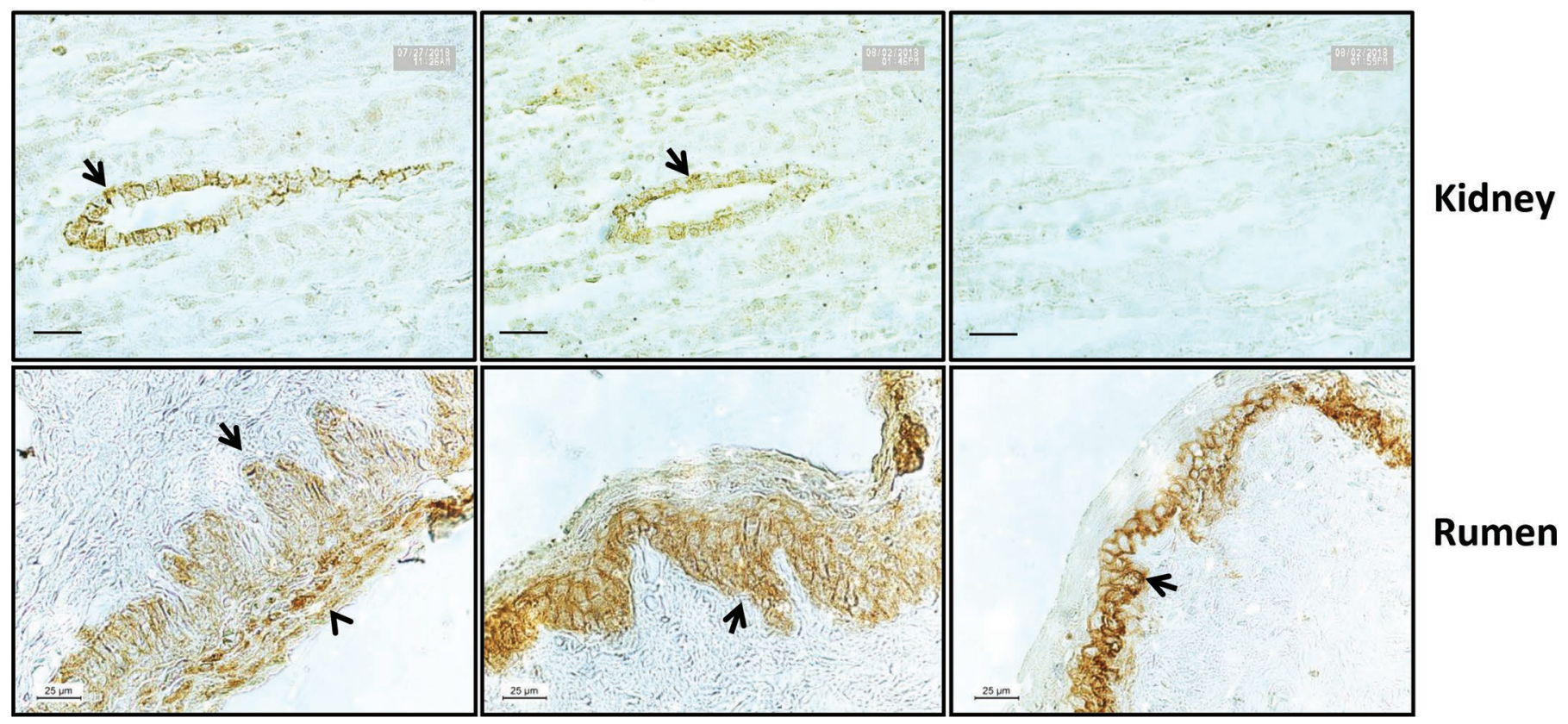

Figure 3. Immunolocalization of aquaporin-3 (AQP3) and urea transporter (UT)-B protein in the bovine kidney and dorsal rumen. Using bovine kidney sections, both AQP3-HPA (HPA014924, Sigma, St. Louis, MO) and AQP-SAB (SAB5200111, Sigma) antibodies (1:100) detected signals in the basolateral cell membranes of collecting ducts (arrows). No such signals were detected by UT-B antibody (1:100). Using sections from dorsal rumen epithelium, AQP3-HPA antibody (1:250) detected signals in all the layers of rumen epithelium, especially in the stratum corneum (arrowhead). In contrast, both AQP3-SAB (1:250) and UT-B antibody (1:500) mainly detected strong cell membrane staining in stratum basale cells (arrows). Scale bar $=25 \mu \mathrm{m}$. 


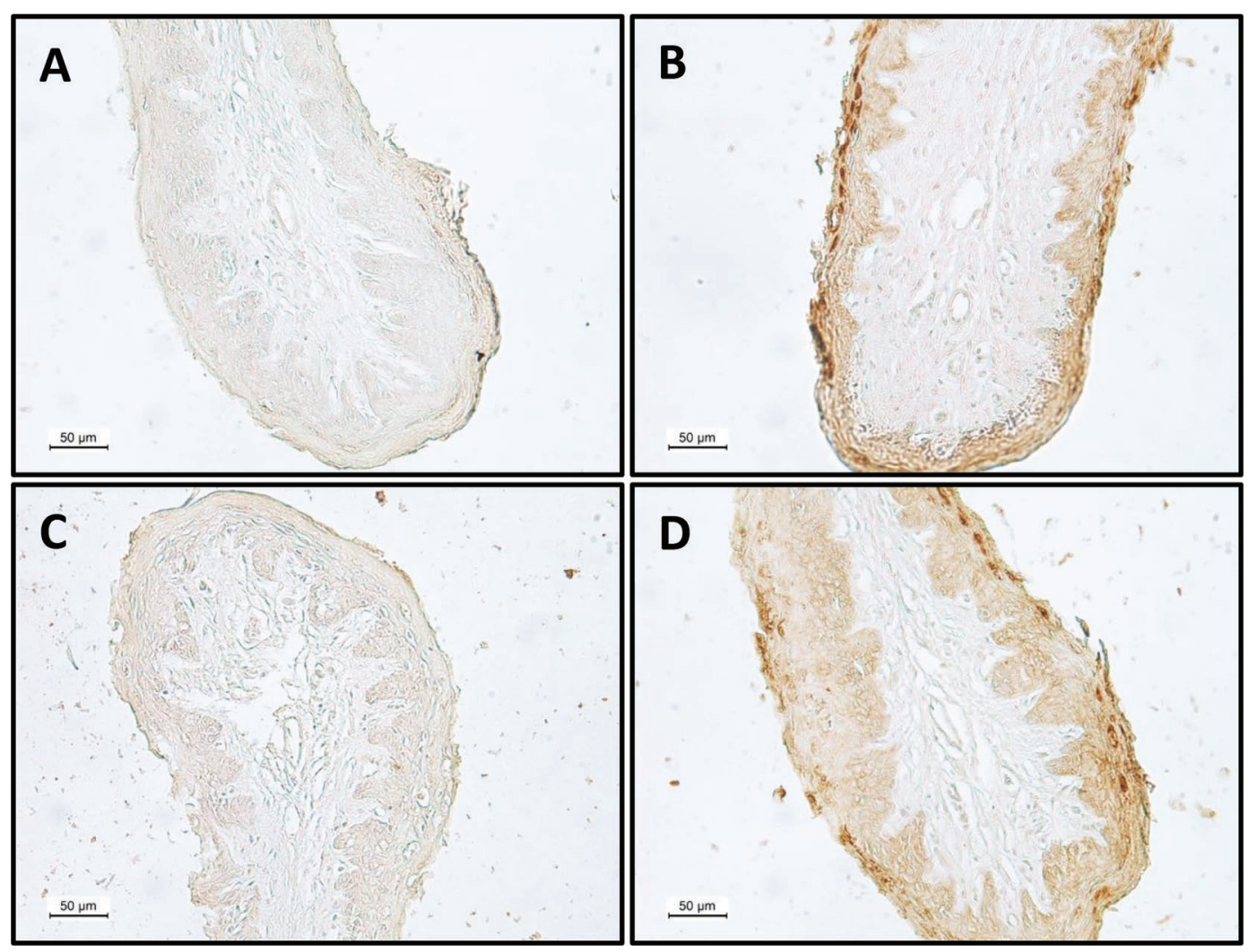

Figure 4. Peptide blocking experiments showing the specificity of aquaporin-3 (AQP3)-HPA (HPA014924, Sigma, St. Louis, MO) antibody. (A) In the absence of primary antibody, no significant staining was observed in the rumen papillae (cranial sac). (B) Overnight incubation with AQP3 antibody (1:250) produced strong signals in stratum corneum and weaker signals in other layers. (C) Pre-incubation of AQP3 antibody with its specific peptide ( $1 \mu \mathrm{g}$ of immunizing peptide $/ 1 \mu \mathrm{L}$ of antibody) prevented most staining. (D) In direct contrast, pre-incubation of AQP3-HPA with the same concentration of a nonspecific peptide $(1 \mu \mathrm{g}$ of UT-A urea transporter peptide/1 $\mu \mathrm{L}$ of antibody) had minimal effect on the staining. Scale bar $=50 \mu \mathrm{m}$.

in the rumen were all specific, as demonstrated by peptide inhibition experiments (Figure 4). Here, the strong staining produced by AQP-HPA (Figure 4B) was almost completely ablated by pre-incubation of the antibody with its original, specific immunizing peptide (Figure 4C). In contrast, no effect occurred after preincubation of the antibody with a similar amount of a nonspecific, unrelated peptide (Figure 4D). These data therefore suggest that the AQP3-HPA staining in all rumen epithelial layers does indeed represent bovine AQP3 protein.

Finally, we have also previously reported that UT-B urea transporters are unexpectedly found within the stratum corneum, in the rumen of cattle fed concentrate diets (Simmons et al., 2009). Taken together with the AQP3 presence in this cell layer reported now in this study, we suggest that there may be another physiological reason for urea transporters in these cells, one that is separate to the trans-epithelial urea transport required by the UNS process. Aquaporin-3 has previously been shown to be vital for hydration in the stratum corneum cells of the skin (Ma et al., 2002). Could this maybe help explain the AQP3 ruminal localization pattern we have detected? Further experimental investigation is now required to answer this intriguing question.

\section{CONCLUSIONS}

Initially, our study confirmed the expression of a single AQP3 transcript in bovine rumen and kidney. Nevertheless, using 2 different antibodies, 2 core AQP3 proteins were detected at 25 and $32 \mathrm{kDa}$, respectively, potentially due to alternative translation initiation sites. Bovine AQP3 protein appeared to be localized to all layers of the rumen epithelium, but the precise physiological significance of this AQP3 distribution pattern remains unclear. Further investigations should now focus on which, if any, of these AQP3 proteins are associated with regulated trans-epithelial urea transport in the bovine rumen. By answering this question, we will gain further insight into the precise role AQP3 transporters play in the crucial UNS process. 


\section{ACKNOWLEDGMENTS}

Chongliang Zhong holds a PhD scholarship jointly financed by the China Scholarship Council (China) and University College Dublin (Ireland). The authors have not stated any conflicts of interest.

\section{REFERENCES}

Abdoun, K., F. Stumpff, I. Rabbani, and H. Martens. 2010. Modulation of urea transport across sheep rumen epithelium in vitro by SCFA and $\mathrm{CO}_{2}$. Am. J. Physiol. Gastrointest. Liver Physiol. 298:G190-G202. https://doi.org/10.1152/ajpgi.00216.2009.

Berends, H., J. J. van den Borne, B. A. Røjen, J. van Baal, and W. J. Gerrits. 2014. Urea recycling contributes to nitrogen retention in calves fed milk replacer and low-protein solid feed. J. Nutr. 144:1043-1049. https://doi.org/10.3945/jn.114.191353.

Coyle, J., S. McDaid, C. Walpole, and G. S. Stewart. 2016. UT-B urea transporter localization in the bovine gastrointestinal tract. J. Membr. Biol. 249:77-85. https://doi.org/10.1007/s00232-015 $-9850-5$.

Ecelbarger, C. A., J. Terris, G. Frindt, M. Echevarria, D. Marples, S. Nielsen, and M. A. Knepper. 1995. Aquaporin-3 water channel localization and regulation in rat kidney. Am. J. Physiol. 269:F663F672. https://doi.org/10.1152/ajprenal.1995.269.5.F663.

Graham, C., and N. L. Simmons. 2005. Functional organization of the bovine rumen epithelium. Am. J. Physiol. Regul. Integr. Comp. Physiol. 288:R173-R181. https://doi.org/10.1152/ajpregu.00425 .2004 .

Kristensen, N. B., A. C. Storm, and M. Larsen. 2010. Effect of dietary nitrogen content and intravenous urea infusion on ruminal and portal-drained visceral extraction of arterial urea in lactating Holstein cows. J. Dairy Sci. 93:2670-2683. https://doi.org/10.3168/ jds.2010-3067.

Lu, Z., F. Stumpff, C. Deiner, J. Rosendahl, H. Braun, K. Abdoun, J. R. Aschenbach, and H. Martens. 2014. Modulation of sheep ruminal urea transport by ammonia and $\mathrm{pH}$. Am. J. Physiol. Regul. Integr. Comp. Physiol. 307:R558-R570. https://doi.org/10.1152/ ajpregu.00107.2014.

Ma, T., M. Hara, R. Sougrat, J. M. Verbavatz, and A. S. Verkman. 2002. Impaired stratum corneum hydration in mice lacking epidermal water channel aquaporin-3. J. Biol. Chem. 277:17147-17153. https://doi.org/10.1074/jbc.M200925200.

Martinotti, S., U. Laforenza, M. Patrone, F. Moccia, and E. Ranzato. 2019. Honey-mediated wound healing: $\mathrm{H}_{2} \mathrm{O}_{2}$ Entry through AQP3 determines extracellular $\mathrm{Ca}^{2+}$ influx. Int. J. Mol. Sci. 20:E764. https://doi.org/10.3390/ijms20030764.

Rojek, A., J. Praetorius, J. Frøkiaer, S. Nielsen, and R. A. Fenton. 2008. A current view of the mammalian aquaglyceroporins. Annu. Rev. Physiol. 70:301-327. https://doi.org/10.1146/annurev.physiol .70 .113006 .100452 .

Røjen, B. A., S. B. Poulsen, P. K. Theil, R. A. Fenton, and N. B. Kristensen. 2011. Short communication: Effects of dietary nitrogen concentration on messenger RNA expression and protein abundance of urea transporter-B and aquaporins in ruminal papillae from lactating Holstein cows. J. Dairy Sci. 94:2587-2591. https:// doi.org/10.3168/jds.2010-4073.

Roudier, N., P. Ripoche, P. Gane, P. Y. Le Pennec, G. Daniels, J. P. Cartron, and P. Bailly. 2002. AQP3 deficiency in humans and the molecular basis of a novel blood group system, GIL. J. Biol. Chem. 277:45854-45859. https://doi.org/10.1074/jbc.M208999200.

Rubenwolf, P. C., W. Otto, S. Denzinger, F. Hofstädter, W. Wieland, and N. T. Georgopoulos. 2014. Expression of aquaporin water channels in human urothelial carcinoma: Correlation of AQP3 expression with tumour grade and stage. World J. Urol. 32:991-997. https://doi.org/10.1007/s00345-013-1153-9.

Saccà, E., M. Corazzin, F. Giannico, C. Fabro, F. Mason, and M. Spanghero. 2018. Effect of dietary nitrogen level and source on mRNA expression of urea transporters in the rumen epithelium of fattening bulls. Arch. Anim. Nutr. 72:341-350. https://doi.org/10 .1080/1745039X.2018.1507977.

Simmons, N. L., A. S. Chaudhry, C. Graham, E. S. Scriven, A. Thistlethwaite, C. P. Smith, and G. S. Stewart. 2009. Dietary regulation of ruminal bovine UT-B urea transporter expression and localization. J. Anim. Sci. 87:3288-3299. https://doi.org/10.2527/jas.2008 -1710 .

Spector, D. A., J. B. Wade, R. Dillow, D. A. Steplock, and E. J. Weinman. 2002. Expression, localization, and regulation of aquaporin-1 to -3 in rat urothelia. Am. J. Physiol. Renal Physiol. 282:F1034F1042. https://doi.org/10.1152/ajprenal.00136.2001.

Stewart, G. S., C. Graham, S. Cattell, T. P. Smith, N. L. Simmons, and C. P. Smith. 2005. UT-B expressed in bovine rumen: Potential role in ruminal urea transport. Am. J. Physiol. Regul. Integr. Comp. Physiol. 289:R605-R612. https://doi.org/10.1152/ajpregu .00127 .2005 .

Stewart, G. S., and C. P. Smith. 2005. Urea nitrogen salvage mechanisms and their relevance to ruminants, non-ruminants and man. Nutr. Res. Rev. 18:49-62. https://doi.org/10.1079/NRR200498.

Walpole, C., A. Farrell, A. McGrane, and G. S. Stewart. 2014. Expression and localization of a UT-B urea transporter in the human bladder. Am. J. Physiol. Renal Physiol. 307:F1088-F1094. https:/ /doi.org/10.1152/ajprenal.00284.2014.

Walpole, M. E., B. L. Schurmann, P. Gorka, G. B. Penner, M. E. Loewen, and T. Mutsvangwa. 2015. Serosal-to-mucosal urea flux across the isolated ruminal epithelium is mediated via urea transporter-B and aquaporins when Holstein calves are abruptly changed to a moderately fermentable diet. J. Dairy Sci. 98:1204-1213. https:// doi.org/10.3168/jds.2014-8757.

Zeuthen, T., and D. A. Klaerke. 1999. Transport of water and glycerol in aquaporin 3 is gated by H. J. Biol. Chem. 274:21631-21636. https://doi.org/10.1074/jbc.274.31.21631.

\section{ORCIDS}

Chongliang Zhong ๑ https://orcid.org/0000-0001-6937-624X Gavin S. Stewart @ https://orcid.org/0000-0001-8688-6401 